\title{
Introduction
}

\section{Balance implants or external prostheses: Which is the wave of the future?}

\author{
John H. J. Allum*
}

${ }^{\mathrm{a}}$ Department of ORL, Division of Audiology and Neurootology, University of Basel Hospital, Basel, Switzerland

Balance impairments affect a large population of individuals especially the elderly, in addition to those with vestibular deficits, CNS disorders, peripheral lower-leg neuropathies, and stroke. When vestibular or proprioceptive function is deficient, essential tasks such as postural control and gaze stabilization are not performed normally. Furthermore, the quality of life of patients is significantly impaired. Recognizing the reduced quality of life, increased financial costs, and increased mortality risk associated with falls, a major scientific effort is underway to determine which of two new techniques holds the most promise of significantly reducing fall risk: vestibular implants or wearable external sensory augmentation devices. Both techniques aim to supplement or replace the vestibular system, either directly or via sensory augmentation or substitution, as this system plays a crucial role in the control of balance. Regardless of the effectiveness of these systems, both have to be measured against the effects of focused balance training alone.

The questions that need to be answered about these systems are similar to those of cochlear implants three decades ago: which technique will improve quality of life most cost-effectively? Then the discussion was between cochlear implants or vibrotactile feedback of sound via a wristband. While an artificial vestibular implant system might be the appropriate solution for many persons with balance impairments, such systems are just on the cusp of clinical reality. A few patients with total vestibular loss have received implants very similar to cochlear implants, but with the capability of restoring vestibulo-ocular function

\footnotetext{
*Corresponding author: John H. J. Allum, Department of ORL, Division of Audiology and Neurootology, University Hospital of Basel, Basel, Switzerland. Tel.: +41 61265 2041; E-mail: john.allum@usb.ch.
}

using head mounted gyroscopes to drive vestibular nerve stimulation. Others consider that elderly fallers may be better served by external wearable aids that provide vibrotactile feedback of body sway to help control balance, typically at the waist or head but also at the upper trunk. Further, these feedback systems may provide persistent carry-over improvements in balance control even weeks after an intense training program. As mentioned above, these devices as well as implants have to be measured against training effects alone. Several authors argue that it may prove more cost effective to reduce anxiety and provide training in fall-avoidance strategies during walking as a form of "inoculation" against future falls.

In this set of papers we have brought together leaders in the field to share their research and views with the aim of sparking discussions and debate on the issues involved with each device. Ideally, new directions for future research will emerge to address several questions addressed in these papers as well as the following crucial questions:

1. Which technique is likely to be more effective in reducing falls and why?

2. What are the advantages and disadvantages for the user and the training institution for each system?

3. Do both systems achieve balance improvements in stance and gait?

4. How can carry-over effects after training with vibrotactile feedback be optimized?

5. Is there a positive after-effect when the implant is switched off?

6. Can implants restore vestibulo-spinal as well as vestibulo-ocular function?

7. Can vibro-tactile feedback improve vestibuloocular function? 